\title{
Identification of Risk Factors For Mortality and Delayed Oral Dietary Intake In Patients With Open Drainage Due To Deep Neck Infections: A Nationwide Study Using A Japanese Inpatient Database
}

Hiroshi Hidaka ( $\sim$ zay00015@nifty.com )

Kansai Medical Unversity https://orcid.org/0000-0001-5214-3159

\section{Kunio Tarasawa}

Department of Health Administration and Polocy, Tohoku University Graduate School of Medicine

Kenji Fujimori

Department of Health Administration and Policy, Tohoku University Graduate School of Medicine

\section{Taku Obara}

Tohoku Medical Megabank Organization

\section{Kiyohide Fushimi}

Department of Health Policy and Informatics, Tokyo Medical and Dental University Graduate School of Medicine

\section{Masafumi Sakagami}

Dep. of Otolaryngology-HNS, Kansai Medical University

Masao Yagi

Dep. of Otolaryngology-HNS, Kansai Medical University

Hiroshi Iwai

Dep. of Otolaryngology-HNS, Kansai Medical University

\section{Research}

Keywords: deep neck infection, abscess, descending necrotizing mediastinitis, mortality, oral intake, DPC database, logistic regression analysis, odds ratio

Posted Date: October 22nd, 2020

DOI: https://doi.org/10.21203/rs.3.rs-94379/v1

License: (a) (1) This work is licensed under a Creative Commons Attribution 4.0 International License. Read Full License 


\section{Abstract}

Backgrounds: Data on risk factors for deep neck infection (DNI) including descending necrotizing mediastinitis (DNM) have been limited. Using a nationwide database, the aim was to identify the factors related to patient death and delay in recovering oral intake.

Methods: We retrospective reviewed a Japanese inpatient database between 2012 and 2017, and data of 4,949 patients with DNM and DNI were extracted. The main outcome was survival at discharge. In a subgroup analysis of the 4,791 patients with survival at discharge, the second outcome was delay in the interval between admission and full recovery of oral intake. In both of the setting, logistic regression analyses were conducted to determine the risk factors.

Results: Regarding survival at discharge, logistic regression analysis showed that age ( $\geq 75$ years), comorbid diabetes mellitus, sepsis, receiving mechanical ventilation, and duration of empirical antibiotic therapy were significantly associated with mortality [adjusted-odds ratios (ORs) (95\% confidence intervals (Cls)] as follows: 5.57 (2.80-11.1), 2.47 (1.69-3.62), 3.32 (2.29-4.82), 3.96 (2.51-6.23), and 1.00 (1.001.02), respectively. In a subgroup analysis of the 4,791 patients with survival at discharge, logistic regression analysis showed the following factors to be significantly associated with delayed oral dietary intake ( $>9$ days), as follows [adjusted OR ( $95 \% \mathrm{Cls})$ ]: age $\geq 75$ years 1.89 (1.48-2.41); body mass index $(\mathrm{BMI})<18.5 \mathrm{~kg} / \mathrm{m}^{2} 1.39$ (1.13-1.71); DNM 1.41 (1.04-1.92); repeated surgical interventions 1.70 (1.162.48); tracheotomy 1.70 (1.44-2.00); mechanical ventilation 1.92 (1.53-2.41); intensive care unit (ICU) admission 1.54 (1.22-1.93); rehabilitation for oral dietary intake 2.05 (1.44-2.92); and duration of empirical antibiotic therapy 1.18 (1.17-1.20).

Conclusions: In this study, a few factors (advanced age, ventilation) were associated with both mortality and delayed oral dietary intake. Conversely, several factors including DNM and repeated surgery were significantly related only to delayed oral dietary intake. Although DNM was not necessarily related to mortality, patients with DNM should receive postoperative care with careful attention to avoid delayed oral dietary intake.

\section{Background}

A deep neck infection (DNI) is a serious disorder that often spreads to other organs and sometimes proves fatal. The most crucial initial treatment strategy in the management of deep neck infections is prompt assessment and selection of patients requiring surgical intervention for drainage. Descending necrotizing mediastinitis (DNM) originating from a deep neck infection is a relatively rare, but rapidly progressive, destructive disease that is often fatal (1-4). Despite improvements in diagnostic methods, surgical technique, and intensive care protocols, DNM has been reported to be a life-threatening condition with mortality rates up to $40 \%(5-7)$. Although the aetiology and clinical outcomes of treating DNI and/or DNM have been extensively reported, no study, other than a few sporadic case reports published in the Japanese language, has addressed dysphagia after surgical treatment (8-10). We thus recently reported 
a logistic regression analysis addressing risk factors related to swallowing recovery after surgical intervention for DNI in a retrospective cohort of 123 patients (11). However, these studies have several limitations attributed to their relatively small sample size from a single medical centre. Moreover, the infrequency of DNM has been one reason for the difficulties in prospective investigations of these patients; no precise therapeutic schema has been proposed, and the optimal treatment for DNM is still debated.

The present study used a nationwide inpatient database in Japan to investigate the factors associated with mortality and delayed recovery of oral dietary intake in patients who underwent drainage surgery for DNI, including DNM.

\section{Methods}

\section{Data source}

Data were extracted from the Diagnostic Procedure Combination (DPC) database, a national inpatient database in Japan. The details of the DPC database have been described previously $(12,13)$. Briefly, the DPC database includes administrative claims data and detailed medical data, collected for all inpatients discharged from participating hospitals. The number of hospitals that participated in the DPC projects included more than $80 \%$ of acute inpatient care in Japan (12-16). To optimize the accuracy of recorded diagnoses, responsible physicians are obligated to record the diagnosis with reference to the medical charts. The dates of hospital admission, surgery, discharge, bedside procedures, and drugs administered are recorded using a uniform data submission format (13).

All patient identifiers were removed from this database. Because of the anonymous nature of the data, the need for informed consent was waived. Study approval was obtained from the Institutional Review Board of the Tokyo Medical and Dental University (protocol Number: M2000-788-15).

\section{Patient Selection And Characteristics}

From the 38.2 million inpatients in the DPC database over a total of 5 years (between April 2012 and March 2017), patients satisfying the following inclusion criteria according to surgical interventions encoded with original Japanese codes $(17,18)$ were selected: 1$)$ underwent incisional drainage for deep neck infection (Japanese code: K384-2) or retropharyngeal abscess (Japanese code: K387, corresponding to intra-pharyngeal drainage for DNI) and 2) underwent mediastinotomy (Japanese code: K502).

The following background characteristics of the patients were assessed: age; sex; body mass index (BMI); smoking status (current or ex-smoker, non-smoker); main diagnosis; comorbidities at admission complications including diabetes mellitus (DM; ICD-10 codes: E10-E14) and sepsis (ICD-10 codes: A40A41, D65) classified according to the International Classification of Diseases, 10th Revision (ICD-10) and 
test data in Japan (12-16); number of surgical interventions; daily records of drug administration and devices used, including dysphagia rehabilitation; length of stay; and discharge status.

According to the World Health Organization and previous reports using the DPC database $(17,18)$, BMI was classified as underweight $\left(<18.5 \mathrm{~kg} / \mathrm{m}^{2}\right)$, normal $\left(18.5 \leq\right.$ to $\left.<30 \mathrm{~kg} / \mathrm{m}^{2}\right)$, and obese $\left(\geq 30 \mathrm{~kg} / \mathrm{m}^{2}\right)$. In addition to these groups, the 774 cases with missing values for calculation of BMI were categorized into the "missing" group. There were also missing values for smoking status (337 cases), and patients were thus subdivided into the following 3 groups: smoking, non-smoking, and missing.

\section{Outcome Measurement}

The primary outcome was survival at discharge. Regarding the subgroup analysis focusing on patients who were alive at discharge, the secondary outcome was the interval between admission and presumed full recovery of oral intake without nutrition from tube feeding or intravenous hydration, according to our previous retrospective, cohort study (11).

\section{Statistical Analysis}

Differences in continuous variables and frequencies between the groups were determined using the Kruskal-Wallis test and the $\chi^{2}$ test, respectively. Multiple logistic regression analysis was performed to identify risk factors associated with survival at discharge (primary outcome) and delayed recovery of postoperative swallowing (secondary outcome). In terms of the secondary outcome, patients were divided into two groups according to median duration (9 days) of recovering oral intake without support of tube feeding or intravenous hydration. Inclusion of independent variables in the model was based on earlier research and existing knowledge (19) in terms of the following risk factors for aggravation of DNI (11, 20-23): age, sex, obesity, smoking habits, DNM, comorbid DM and sepsis, repeated surgical interventions, undergoing tracheotomy and/or mechanical ventilation, and duration of empirical antibiotic therapy. A $p$ value $<0.05$ was considered significant. All statistical analyses were conducted using EZR software (24).

\section{Results}

\section{Demographic Data}

A total of 6,405 cases were identified, as shown in Fig. 1. Of the 6,040 patients who underwent drainage for a deep neck infection and/or retropharyngeal abscess, 13 and 1,232 cases were excluded because their surgeries were attributed to an injury or foreign body and related to resection of pharyngeal tumour or haemostasis after laryngopharyngeal surgeries, respectively. Of the 365 patients who underwent mediastinotomy, 154 patients were selected after excluding 108 patients who underwent surgery for resection of a mediastinal tumour, 6 patients with mediastinitis attributed to injury or a foreign body, and 
97 patients whose mediastinitis was attributed to a thoracic lesion including the oesophagus, because ICD-10 codes addressing acute infections in the head and neck region were lacking. Finally, 4,949 patients from 635 hospitals were selected.

Of all of the 4,949 patients, the 4,791 patients who were alive at discharge were divided into 2 groups according to the median interval ( 9 days) between admission and full recovery of oral intake without nutrition from tube feeding or intravenous hydration. Namely, group A and group B included the cases with intervals less than $<10$ days and $\geq 10$ days, respectively. Another 158 patients who died in hospital were assigned to group $\mathrm{C}$.

Table 1 summarizes the baseline characteristics of each group of patients. The patients were categorized into three groups according to the clinical outcome, as follows: A) alive at discharge undergoing nutritional support for 9 days after admission; $B$ ) alive at discharge undergoing nutritional support for more than 10 days after the first operation; and $C$ ) died in hospital. The median ages of groups $A, B$, and $C$ were 56,66 , and 73 years, respectively. Group $C$ showed a significantly higher interquartile range for age (19-96 years) than the other groups ( $p<0.001$, Kruskal-Wallis test). Conversely, both the male to female ratios and obesity evaluated by the BMI of the three groups were not significantly different. Smoking status was also not significantly different. 
Table 1

Baseline characteristics of patients categorized by survival at discharge and prolonged nutritional support by tube feeding or intravenous hydration

\section{Group}

$$
A(N=2499) \quad B(N=2292) \quad C(N=158) \quad P \text { value }
$$

Age (y)

$<0.001^{\star \star}$

Median

Range

Interquartile range

Sex

Male

Female

Obesity $\left(\mathrm{BMl}, \mathrm{kg} / \mathrm{m}^{2}\right)$

$<18.5$

$\geq 18.5$ to $<30$

$\geq 30$

Unknown

Diabetes mellitus

No

Yes

Smoking

No

Yes

Unknown

Descending necrotizing mediastinitis
56

$0-99$

$34.0-69.0$

$51.8-76$

0.23

$1562(62.5 \%) \quad 1405(61.3 \%) \quad 107(67.7 \%)$

$937(37.5 \%) \quad 887(38.7 \%) \quad 51(32.3 \%)$

\begin{tabular}{lll}
\hline $72(18.9 \%)$ & $383(16.7 \%)$ & $23(14.6 \%)$ \\
\hline $772(70.9 \%)$ & $1631(71.2 \%)$ & $114(72.2 \%)$ \\
$101(4.0 \%)$ & $109(4.8 \%)$ & $7(4.4 \%)$ \\
\hline $54(6.2 \%)$ & $169(7.4 \%)$ & $14(8.9 \%)$ \\
\hline $981(79.3 \%)$ & $1365(60.0 \%)$ & $48(30.4 \%)$ \\
\hline $518(20.7 \%)$ & $927(40.0 \%)$ & $110(69.6 \%)$
\end{tabular}

$0.26^{\#}$

$<0.001$

$0.19^{\#}$

\begin{tabular}{lll}
$1340(53.6 \%)$ & $1202(52.4 \%)$ & $68(43.0 \%)$ \\
\hline $804(32.2 \%)$ & $705(30.8 \%)$ & $56(35.4 \%)$ \\
\hline $355(14.2 \%)$ & $385(16.8 \%)$ & $34(21.5 \%)$
\end{tabular}

$<0.001$

Patients were categorized into three groups according to outcomes related to survival at discharge and prolonged interval for achieving oral intake, as follows: A) alive at discharge undergoing nutrition support for 9 days after admission; B) alive at discharge undergoing nutrition support for more than 10 days after the first operation; and C) died in hospital.

\#Chi-squared test, conducted for other than unknown cases for each characteristic.

**Kruskal-Wallis test at $\mathrm{P}<.001$. 


\begin{tabular}{|c|c|c|c|c|}
\hline & Group & & & \\
\hline No & $2409(94.4 \%)$ & $1878(81.9 \%)$ & $112(70.9 \%)$ & \\
\hline Yes & $90(3.6 \%)$ & $414(18.1 \%)$ & $46(29.1 \%)$ & \\
\hline Sepsis & & & & $<0.001$ \\
\hline No & $2370(94.8 \%)$ & $1841(80.3 \%)$ & $77(48.7 \%)$ & \\
\hline Yes & $129(5.2 \%)$ & $451(19.7 \%)$ & $81(51.3 \%)$ & \\
\hline Surgical intervention / More than once & & & & $<0.001$ \\
\hline No & $2453(98.2 \%)$ & $1983(86.5 \%)$ & $140(88.6 \%)$ & \\
\hline Yes & $46(1.8 \%)$ & $309(13.5 \%)$ & $18(11.4 \%)$ & \\
\hline Tracheotomy & & & & $<0.001$ \\
\hline No & $2026(81.1 \%)$ & $1360(59.3 \%)$ & $93(58.9 \%)$ & \\
\hline Yes & $473(18.9 \%)$ & $932(40.7 \%)$ & $65(41.1 \%)$ & \\
\hline Mechanical ventilator & & & & $<0.001$ \\
\hline No & $2250(90.0 \%)$ & $1416(61.8 \%)$ & $81(51.3 \%)$ & \\
\hline Yes & $249(10.0 \%)$ & $876(38.2 \%)$ & $81(51.3 \%)$ & \\
\hline Intensive care unit admission & & & & $<0.001$ \\
\hline No & $2258(90.4 \%)$ & $1556(67.9 \%)$ & $79(50.0 \%)$ & \\
\hline Yes & $241(9.6 \%)$ & $736(32.1 \%)$ & $79(50.0 \%)$ & \\
\hline Rehabilitation for oral dietary intake & & & & $<0.001$ \\
\hline No & $2445(97.8 \%)$ & $1968(85.9 \%)$ & $142(89.9 \%)$ & \\
\hline Yes & $54(2.2 \%)$ & $324(14.1 \%)$ & $16(10.1 \%)$ & \\
\hline Duration of antibiotic therapy (days) & & & & $<0.001^{* \star}$ \\
\hline Median & 9 & 18 & 20 & \\
\hline Range & $1-75$ & $1-311$ & $1-199$ & \\
\hline
\end{tabular}

Patients were categorized into three groups according to outcomes related to survival at discharge and prolonged interval for achieving oral intake, as follows: A) alive at discharge undergoing nutrition support for 9 days after admission; B) alive at discharge undergoing nutrition support for more than 10 days after the first operation; and C) died in hospital.

\#Chi-squared test, conducted for other than unknown cases for each characteristic.

$\star \star$ Kruskal-Wallis test at $\mathrm{P}<.001$. 


\begin{tabular}{|l|l|}
\hline \multicolumn{1}{|c|}{ Group } \\
\hline Interquartile range & $7-12$ \\
\hline $\begin{array}{l}\text { Patients were categorized into three groups according to outcomes related to survival at discharge } \\
\text { and prolonged interval for achieving oral intake, as follows: A) alive at discharge undergoing nutrition } \\
\text { support for } 9 \text { days after admission; B) alive at discharge undergoing nutrition support for more than } \\
10 \text { days after the first operation; and C) died in hospital. }\end{array}$ \\
\hline \#Chi-squared test, conducted for other than unknown cases for each characteristic. \\
\hline$* \star$ Kruskal-Wallis test at $\mathrm{P}<.001$. \\
\hline
\end{tabular}

Concerning disease comorbidity, the prevalence of DM was higher in group $\mathrm{C}(69.6 \%)$ than in group $\mathrm{A}$ $(20.7 \%)$ and group $B(40.0 \%)(p<0.001)$. In addition, comorbid sepsis was significantly higher in group $C$ $(51.3 \%)$ than in group $A(5.2 \%)$ and group $B(19.7 \%)(p<0.001)$. In terms of the presence of DNM, the prevalence was higher in group $C(29.1 \%)$ than in group A (3.6\%) and group B $(18.1 \%)(p<0.001)$.

Regarding surgical interventions, the prevalence of repeated surgical interventions (more than one surgical drainage) was higher in both group B (13.5\%) and group C $(11.4 \%)$ than in group A $(1.8 \%)(p<$ 0.001). The prevalence of subsequent tracheotomy was greater than $40 \%$ in both group $B$ and group $C$, significantly higher than in group A $(18.9 \%)(p<0.001)$. Moreover, there was a significant difference in patients undergoing mechanical ventilation and/or intensive care unit (ICU) therapy among the groups ( $p$ $<0.001)$.

As for postoperative care, the prevalence of receiving rehabilitation therapy for oral dietary intake was lower in group $A$ than in group $B(p<0.001)$. Regarding the duration of antibiotic therapy, the mean durations of groups $A, B$, and $C$ were 9,18 , and 20 days, respectively $(p<0.001$, Kruskal-Wallis test).

\section{Risk Factors Contributing To In-hospital Mortality}

Logistic regression analysis showed that the following factors were significant (with crude odds ratios (ORs)): age $\geq 50$ years 5.31 [95\% confidence interval $(\mathrm{Cl}), 2.82-10.0$ ]; age $\geq 75$ years $8.88(95 \% \mathrm{Cl} 4.66-$ 16.9); DM 5.31 (95\% Cl 3.76-7.49); DNM 3.49 (95\%Cl 2.45-4.98); sepsis 7.64 (95\% Cl 5.52-10.6); tracheostomy $1.68(95 \% \mathrm{Cl} 1.22-2.33)$; mechanical ventilation $8.72(95 \% \mathrm{Cl} 6.1-12.4)$; ICU admission 3.9 $(95 \% \mathrm{Cl} 2.84-5.37)$; and duration of intravenous antibiotic therapy $1.02(95 \% \mathrm{Cl} 1.02-1.03)$ (Table 2). Of them, the following factors were significant, with ORs adjusted by the other variables (adjusted ORs): age $\geq 50$ years $2.96(95 \% \mathrm{Cl} 1.51-5.80)$; age $\geq 75$ years 5.57 (95\% Cl 2.80-11.1); comorbid DM 2.47 (95\%Cl 1.69-3.62); sepsis; 3.32 (95\% Cl 2.29-4.82); and mechanical ventilation 3.96 (95\% Cl 2.51-6.23). 
Table 2

Multiple logistic regression analysis of risk factors for survival at discharge among patients who underwent surgical intervention for deep neck infections

\begin{tabular}{|lll|}
\hline & $\begin{array}{l}\text { Crude OR } \\
(95 \% \text { Cl) }\end{array}$ & $\begin{array}{l}\text { Adjusted OR } \\
(95 \% \text { Cl) }\end{array}$ \\
\hline Age (y) & & \\
\hline$<50$ & 1.00 & 1.00 \\
\hline $50 \leqq,<75$ & $5.31(2.82-10.0)$ & $2.96(1.51-5.80)$ \\
\hline $75 \leqq$ & $8.88(4.66-16.9)$ & $5.57(2.80-11.1)$ \\
\hline Sex: female (vs. male) & $0.78(0.55-1.09)$ & $0.74(0.46-1.20)$ \\
\hline Obesity: BMI (kg/m $\left.{ }^{2}\right)$ & & \\
\hline $18.5 \leq,<30$ & 1.00 & 1.00 \\
\hline$<18.5$ & $0.80(0.51-1.26)$ & $1.37(0.83-2.26)$ \\
\hline$\geq 30$ & $1.00(0.46-2.16)$ & $0.78(0.34-1.79)$ \\
\hline Diabetes mellitus & $5.31(3.76-7.49)$ & $2.47(1.69-3.62)$ \\
\hline Smoking & $1.39(0.97-1.99)$ & $1.19(0.78-1.83)$ \\
\hline Descending necrotizing mediastinitis & $3.49(2.45-4.98)$ & $1.14(0.76-1.73)$ \\
\hline Sepsis & $7.64(5.52-10.6)$ & $3.32(2.29-4.82)$ \\
\hline Surgical intervention / more than once & $1.61(0.97-2.66)$ & $0.60(0.35-1.05)$ \\
\hline Tracheotomy & $1.68(1.22-2.33)$ & $0.95(0.66-1.35)$ \\
\hline Mechanical ventilation & $8.72(6.10-12.4)$ & $3.96(2.51-6.23)$ \\
\hline Intensive care unit admission & $3.90(2.84-5.37)$ & $1.22(0.83-1.79)$ \\
\hline Rehabilitation for oral dietary intake & $1.32(0.78-2.23)$ & $0.42(0.24-0.73)$ \\
\hline Duration of antibiotic therapy (days) & $1.02(1.02-1.03)$ & $1.00(1.00-1.02)$ \\
\hline Cl = confidence interval; OR = odds ratio. & & \\
\hline & & \\
\hline & & \\
\hline & & \\
\hline
\end{tabular}

\section{Interval Between Admission And Achievement Of Oral Intake}

Table 3 shows the distribution of intervals between admission and achievement of oral intake in 4,791 patients who were alive at discharge (groups A and B in Table 1) categorized by DNM, which represents 
subgroup analyses without group C. More than half of the 4,287 patients without DNM (56.2\%) achieved oral dietary intake $<10$ days after admission. Conversely, the rate was only $17.9 \%$ in the 504 patients with DNM. In addition, $57.7 \%$ of the patients with DNM could not achieve oral dietary intake in 20 days.

Table 3

Interval between admission and achievement of oral intake without nutritional support from tube feeding or intravenous hydration

\begin{tabular}{|lll|}
\hline \multicolumn{3}{|c|}{ Group } \\
\hline Interval (days) & DNM - & DNM + (N=504) \\
\hline $0-4$ & $(\mathrm{~N}=4287)$ & $28(5.6 \%)$ \\
\hline $5-9$ & $1100(25.9 \%)$ & $62(12.3 \%)$ \\
\hline $10-15$ & $1299(30.3 \%)$ & $66(13.1 \%)$ \\
\hline $16-20$ & $671(15.7 \%)$ & $57(11.3 \%)$ \\
\hline$>20$ & $339(7.9 \%)$ & $291(57.7 \%)$ \\
\hline $\begin{array}{l}\text { Patients were categorized into groups according to deep neck infection (DNI) with or without } \\
\text { descending necrotizing mediastinitis (DNM). }\end{array}$ \\
\hline
\end{tabular}

In addition, logistic regression analysis showed that sex, obesity, and smoking did not differ according to the delay in oral dietary intake (Table 4). Conversely, the following factors were significant: age $\geq$ 50 years $2.06(95 \% \mathrm{Cl} 1.80-2.35)$; age $\geq 75$ years 3.37 (95\% Cl 2.86-3.97); DM $2.60(95 \% \mathrm{Cl} 2.29-2.95)$; DNM 5.90 (95\% Cl 4.66-7.47); sepsis 4.50 (95\% Cl 3.67-5.52); repeated surgical interventions $8.31(95 \% \mathrm{Cl}$ 6.06-11.4); tracheostomy $2.94(95 \% \mathrm{Cl} 2.58-3.34)$; mechanical ventilation $5.59(95 \% \mathrm{Cl} 4.78-6.53)$; ICU admission 4.43 (95\% Cl 3.78-5.20); rehabilitation for oral dietary intake 7.45 (95\% $\mathrm{Cl} 5.55-10.0)$; and duration of intravenous antibiotic therapy $1.22(95 \% \mathrm{Cl} 1.21-1.24)$. Of them, the following factors were significant with their ORs adjusted by the other variables: age $\geq 75$ years 1.89 (95\% $\mathrm{Cl} 1.48-2.41)$; DNM $1.41(95 \% \mathrm{Cl} 1.04-1.92)$; repeated surgical interventions $1.70(95 \% \mathrm{Cl} 1.16-2.48)$; tracheotomy $1.70(95 \% \mathrm{Cl}$ 1.44-2.00); mechanical ventilation 1.92 (95\% Cl 1.53-2.41); ICU admission 1.54 (95\% Cl 1.22-1.93); rehabilitation for oral dietary intake 2.05 (95\% $\mathrm{Cl} 1.44-2.92)$; and duration of intravenous antibiotic therapy $1.18(95 \% \mathrm{Cl} 1.17-1.20)$. 
Table 4

Multiple logistic regression analysis of risk factors for a prolonged interval for achieving oral intake among patients who underwent surgical intervention for deep neck infections

\begin{tabular}{|lll|}
\hline & $\begin{array}{l}\text { Crude OR } \\
(95 \% \text { Cl) }\end{array}$ & $\begin{array}{l}\text { Adjusted OR } \\
(95 \% \text { Cl) }\end{array}$ \\
\hline Age (y) & & \\
\hline$<50$ & 1.00 & 1.00 \\
\hline $50 \leq,<75$ & $2.06(1.8-2.35)$ & $1.19(0.97-1.46)$ \\
\hline$\geq 75$ & $3.37(2.86-3.97)$ & $1.89(1.48-2.41)$ \\
\hline Sex: female (vs. male) & $1.05(0.94-1.18)$ & $1.09(0.91-1.31)$ \\
\hline Obesity: BMI (kg/m $\left.{ }^{2}\right)$ & & \\
\hline $18.5 \leq,<30$ & 1.00 & 1.00 \\
\hline$<18.5$ & $0.88(0.76-1.02)$ & $1.39(1.13-1.71)$ \\
\hline$\geq 30$ & $1.17(0.89-1.55)$ & $1.11(0.77-1.59)$ \\
\hline Diabetes mellitus & $2.60(2.29-2.95)$ & $0.97(0.82-1.16)$ \\
\hline Smoking & $0.98(0.86-1.11)$ & $0.92(0.77-1.10)$ \\
\hline Descending necrotizing mediastinitis & $5.90(4.66-7.47)$ & $1.41(1.04-1.92)$ \\
\hline Sepsis & $4.50(3.67-5.52)$ & $1.15(0.88-1.51)$ \\
\hline Surgical intervention / more than once & $8.31(6.06-11.4)$ & $1.70(1.16-2.48)$ \\
\hline Tracheotomy & $2.94(2.58-3.34)$ & $1.70(1.44-2.00)$ \\
\hline Mechanical ventilation & $5.59(4.78-6.53)$ & $1.92(1.53-2.41)$ \\
\hline Intensive care unit admission & $4.43(3.78-5.20)$ & $1.54(1.22-1.93)$ \\
\hline Rehabilitation for oral dietary intake & $7.45(5.55-10.0)$ & $2.05(1.44-2.92)$ \\
\hline Duration of antibiotic therapy (days) & $1.22(1.21-1.24)$ & $1.18(1.17-1.20)$ \\
\hline Cl = confidence interval; OR = odds ratio. & & \\
\hline & & \\
\hline & & \\
\hline
\end{tabular}

\section{Discussion}

In this study, a total of 4,949 patients throughout Japan who underwent drainage surgery for DNIs were investigated using a Japanese nationwide inpatient database. To the best of our knowledge, this is the first study to investigate the factors affecting mortality and delay in oral dietary intake in patients with DNIs, including DNM, in a nationwide clinical setting. The present study was also unique in that it 
compared these two outcomes by logistic regression analysis using the same clinical risk factors as independent variables. Interestingly, a few factors were associated with both in-hospital mortality and delay in oral dietary intake. Comorbid DM and sepsis were significantly associated only with in-hospital mortality. Conversely, several factors (DNM, repeated surgical interventions, ICU admission, and rehabilitation for oral dietary intake) were found to be significantly related only to delayed oral dietary intake.

Advanced age has been reported to be a risk factor for developing DNIs $(25,26)$. The present study showed that age $\geq 75$ years was significantly associated with both mortality (adjusted OR $5.57,95 \% \mathrm{Cl}$ 2.8-11.1) and longer recovery of oral dietary intake (adjusted OR $1.89,95 \% \mathrm{Cl} 1.48-2.41$ ). The presence of systemic disease was the most important predisposing factor for the severity of DNI. Of them, comorbid DM is a well-known risk factor. The present study showed that DM was correlated with mortality, with an adjusted OR of $2.47(95 \% \mathrm{Cl} 1.69-3.62)$. These results are consistent with a recent systematic review and meta-analysis, showing that DM was associated with a higher prevalence of multispace spread of infection, complications, and failure to identify pathogens, with risk ratios of 1.96, 2.42, and 1.29 , respectively (22). Conversely, DM was not a significant factor related to delay in oral dietary intake, with an adjusted OR of $0.97(95 \% \mathrm{Cl} 0.82-1.16)$. No background mechanisms have been confirmed to explain this inconsistency. One hypothesis is that this factor was adjusted by other variables, because the crude OR of this factor was $2.60(95 \% \mathrm{Cl} 2.29-2.95)$.

Similar to sex, obesity evaluated by BMI was relatively uniform among the three groups. Specifically, the prevalence of patients with obesity $\left(\mathrm{BMl} \geq 30 \mathrm{~kg} / \mathrm{m}^{2}\right)$ was $4-5 \%$ in all groups, and it was not a risk factor for either mortality or delay in oral dietary intake. Although the relationship between the severity of DNIs and obesity has yet to be determined, a previous report showed that obesity $\left(\mathrm{BMI} \geq 30 \mathrm{~kg} / \mathrm{m}^{2}\right)$ was correlated with longer hospitalization (27). The DPC database has missing data for evaluating BMI in $7 \%$ (337/4949) of cases. In the DPC data, the codes corresponding to each surgery, clinical procedure, and medication are almost complete because they are compulsory items for reimbursement of healthcare costs defined in a central system in Japan to check the adequacy of the data (All-Japan Federation of the National Health Insurance Organization). However, other information submitted with claims that is not directly related to charges may be missing, such as height/weight and smoking index (28). These less accurate data may also be responsible for the failure to identify obesity and smoking as risk factors for both mortality and delay in oral dietary intake in the present study. The smoking index was missing in $15 \%(754 / 4791)$ of cases in the DPC data, similar to the previous study (28).

The mortality rate for all patients with DNIs was 3\% (158/4949), and that for patients with DNM was $8 \%$ $(46 / 550)$. The latter rate is consistent with recent meta-analyses $(6,7)$ demonstrating mortality of $9-10 \%$ for the combined cervical and transthoracic approach compared to mortality of $47-50 \%$ for cervical drainage only in cases of infection to the inferior mediastinum. Interestingly, comorbidity with DNM was not found to be a significant factor for mortality. These results could be attributed to the spreading of strategies in Japan for transthoracic drainage in patients with DNM with extension below the tracheal bifurcation $(11,29)$. In the present cohort, 22\% $(158 / 550)$ of the patients with DNM underwent 
transthoracic drainage. In contrast to the results for mortality, the present study showed that DNM was a significant factor for the delay in oral dietary intake, with an adjusted OR of 1.41 (95\% Cl 1.04-1.92). In addition to severe infection, systematic debridement and broad opening of the involved fascial planes are considered to contribute to limited laryngeal elevation and/or severe scar contraction specifically around the cricopharyngeal muscle (11). Therefore, patients with DNM should receive postoperative care with careful attention to the risk of delayed recovery of oral intake.

Of the systemic diseases contributing to mortality, several previous reports addressed death due to complications with sepsis $(25,30-33)$. In contrast to the outcome related to DNM, the present study showed that sepsis was a significant factor only for mortality, with an adjusted OR of $3.32(95 \% \mathrm{Cl} 2.29-$ $4.82)$, not for a delay in oral dietary intake, with an adjusted OR of $1.15(95 \% \mathrm{Cl} 0.88-1.51)$. Therefore, sepsis, rather than DNM, should be considered a risk factor for mortality in treating DNIs and DNM.

In the present study, tracheotomy contributed only to a delay in oral dietary intake, with an adjusted OR of $1.70(95 \% \mathrm{Cl} 1.44-2.00)$, which was similar to the result for DNM. Previous reports advocated tracheotomy for DNI cases with severe airway obstruction and/or presumed difficult re-intubation specifically managed by otolaryngologists $(6,34-37)$. These results are consistent with our previous study and imply that a disturbance in swallowing function may be encountered after tracheotomy, presumably resulting from desensitization of the larynx after diversion of the air passage or fixation of the larynx $(11,38)$.

It is plausible that undergoing repeated surgery is related to a delay in oral dietary intake, with an adjusted OR of $1.70(95 \% \mathrm{Cl} 1.16-2.48)$. Interestingly, this factor was opposite to the risk for mortality, with an adjusted OR of 0.45 (95\% Cl 0.23-2.07). Singhal (39) reported that CT imaging of the neck and chest should be performed with any clinical deterioration of the patient or empirically 48-72 hours after an operative drainage procedure to identify any progression of the infection. Because initial drainage is often inadequate in patients specifically with DNM, the present results suggest surgical interventions with repeat drainage. However, these patients should receive postoperative care with careful attention to the risk of delay for recovering oral intake.

Postoperative care has been reported to play a critical role in patients with DNIs and DNM $(5,31,40)$. Appropriate and careful selection of patients for ICU admission is sometimes fundamental for these patients to manage severe sepsis and/or septic shock and every possible complication, both at the beginning and after surgery $(31,40)$. In the present study, ICU admission was not found to be a crucial factor related to mortality. One hypothesis for these results is that the above-mentioned critical care might contribute to the survival of patients with severe morbidities.

In contrast to the above-mentioned risk factor, mechanical ventilation was a significant risk factor for both mortality and delay in oral dietary intake, with adjusted ORs of $3.96(95 \% \mathrm{Cl} 2.51-6.23)$ and 1.92 $(95 \% \mathrm{Cl} 1.53-2.41)$, respectively. One background hypothesis is that the deteriorated conditions of the patients led to endotracheal intubation in the first place. According to the previous support, most cases with DNM showed whole body deterioration and received support from an artificial respirator for an extended period $(2,40)$. Similar to the results for mechanical ventilation, the duration of empirical 
antibiotic therapy (days) was also a significant risk factor for both mortality and delay in oral dietary intake, with adjusted ORs of $1.00(95 \% \mathrm{Cl} 1.00-1.02)$ and $1.18(95 \% \mathrm{Cl} 1.17-1.19)$, respectively. Along with surgical drainage, high-dose intravenous antibiotics are the mainstay for the management of DNIs. These results correspond to the longer intravenous antibiotic therapy in more critically ill patients with infection.

Finally, if patients can recover from their severely ill condition, rehabilitation for dysphagia might be indicated. Although only a few reports have addressed rehabilitation for DNIs or DNM because of the absence of detailed swallowing evaluation $(11,41)$, the present nationwide study showed that approximately $10 \%$ of patients with DNIs received rehabilitation therapy. This was a significant factor for a delay in oral dietary intake, with an adjusted OR of $2.05(95 \% \mathrm{Cl} 1.44-2.92)$. These results correspond to the situation where the right person (suffering from dysphagia) is in the right place (undergoing rehabilitation).

Several limitations of this study need to be acknowledged. First, this study was based on a retrospective cohort study using a national Japanese database, and generalization of the results outside Japan may not be appropriate. Second, comorbidities are less accurately recorded in administrative claims databases than in planned prospective studies. Third, the absence of records on vital signs, blood tests, and blood and bacteriological cultures in the DPC database precluded a more rigorous definition of septic shock, as discussed in the previous report (13). Moreover, DPC data come from an inpatient database, and it is difficult to evaluate the delay from onset to intervention.

Within these limitations, the current study with the largest retrospective cohort using a nationwide database has several advantages. It was found that clinical risk factors differed between mortality and delay in oral dietary intake other than age $\geq 75$ years, sepsis, mechanical ventilation, and duration of empirical antibiotic therapy. Other than these factors, DM contributed only to mortality. Conversely, DNM, repeated surgery, tracheostomy, and intensive care unit admission contributed to a delay in oral dietary intake, but not mortality. Further research including multicentre prospective studies of DNM and DNI is necessary to establish precise therapeutic approaches for managing DNIs and the optimal treatment for DNM.

\section{Conclusions}

In this study using a large nationwide inpatient database, a few factors (advanced age, mechanical ventilation, and duration of empirical antibiotic therapy) were associated with both mortality and delay in oral dietary intake in patients with DNIs including DNM. Comorbid DM and sepsis were significantly associated only with mortality. Conversely, several factors (DNM, repeated surgical interventions, ICU admission, and rehabilitation for oral dietary intake) were found to be significantly related only to delayed oral dietary intake. Although DNM was not necessarily related to mortality, patients with DNM should receive postoperative care with careful attention to avoid a delay in oral dietary intake.

\section{Declarations}


Availability of data and materials: The datasets used and/or analyzed during the current study are available from the corresponding author on reasonable request.

Acknowledgements: None

Contributions: $\mathrm{HH}$ designed and supervised the study. KT ,FK and KF acquired all clinical data. All authors performed data analysis and interpretation. TO, MS and MY assisted in the statistical analyses. $\mathrm{HH}$ and $\mathrm{HI}$ wrote the manuscript. All authors read and approved the final manuscript.

List of abbreviations: deep neck infection (DNI); descending necrotizing mediastinitis (DNM); Diagnostic Procedure Combination (DPC); diabetes mellitus (DM); body mass index (BMI); odds ratio (OR); confidence interval $(\mathrm{Cl})$

Funding: A portion of this study was supported by JSPS KAKENHI Grant-in-Aid No. 20K09699 (to HH) from the Ministry of Education, Science, and Culture of Japan.

\section{Ethics declarations}

Ethics approval and consent to participate: All patient identifiers were removed from this database. Because of the anonymous nature of the data, the need for informed consent was waived. Study approval was obtained from the Institutional Review Board of the Tokyo Medical and Dental University (protocol Number: M2000-788-15).

Consent for publication: Not applicable

Competing interests: The authors declare that they have no competing interests.

\section{References}

1. Estrera AS, Landay MJ, Grisham JM, et al. Descending necrotizing mediastinitis. Surg Gynecol Obstet. 1983;157:545-52.

2. Uwa N, Terada T, Saeki N, et al. Clinical features of 5 patients with descending necrotizing mediastinitis originating from deep neck infection. Auris Nasus Larynx. 2010;37:530-4.

3. Kocher GJ, Hoksch B, Caversaccio M, et al. Diffuse descending necrotizing mediastinitis: surgical therapy and outcome in a single-centre series. Eur J Cardiothorac Surg. 2012;42:e66-72.

4. Ishinaga $\mathrm{H}$, Otsu $\mathrm{K}$, Sakaida $\mathrm{H}$, et al. Descending necrotizing mediastinitis from deep neck infection. Eur Arch Otorhinolaryngol. 2013;270:1463-6.

5. Sandner A, Börgermann J, Kösling S, et al. Descending necrotizing mediastinitis: early detection and radical surgery are crucial. J Oral Maxillofac Surg. 2007;65:794-800.

6. Ridder GJ, Maier W, Kinzer S, et al. Descending necrotizing mediastinitis: contemporary trends in etiology, diagnosis, management, and outcome. Ann Surg. 2010;251:528-34. 
7. Prado-Calleros HM, Jiménez-Fuentes $E$, Jiménez-Escobar I. Descending necrotizing mediastinitis: Systematic review on its treatment in the last 6 years, 75 years after its description. Head Neck. 2016;38(Suppl 1):E2275-83.

8. Hara $\mathrm{H}$, Imate $\mathrm{Y}$, Horiike $\mathrm{O}$, et al. Effectiveness of laryngeal suspension and cricopharyngeal myotomy in treating a swallowing disturbance [in Japanese]. J Jpn Brochoesophagol Soc. 2001;52:414-9.

9. Ishinaga $\mathrm{H}$, Nakamura $\mathrm{S}$, Otsu $\mathrm{K}$, et al. Dysphagia after undergoing treatment for deep neck infection [in Japanese]. Deglutition. 2012;1:338-41.

10. Uemaetomari I, Hayashi K, Takahashi K. Two cases of dysphagia after treating descending necrotizing mediastinitis [in Japanese]. J Jpn Soc Head Neck Surg. 2015;25:103-7.

11. Hidaka $\mathrm{H}$, Ozawa $\mathrm{D}$, Kuriyama $\mathrm{S}$, et al: Risk factors for delayed oral dietary intake in patients with deep neck infections including descending necrotizing mediastinitis. Eur Arch Otolaryngol 2017; 3951-3958.

12. Matsuda J, Fujimori K, Kuwabara K, et al. Diagnostic procedure combination as an infrastructure for the clinical study. Asian Pan J Dis Manage. 2011;5:81-7.

13. Iwagami M, Yasunaga H, Doi K, et al. Postoperative polymyxin B hemoperfusion and mortality in patients with abdominal septic shock: a propensity-matched analysis. Crit Care Med. 2014;42:118793.

14. Miyamoto $\mathrm{Y}$, Aso S, Iwagami M, et al: Association between IV thiamine and mortality in patients with septic shock: A nationwide observational study. Crit Care Med 2020 May 11.

doi:10.1097/CCM.0000000000004394 [Online ahead of print].

15. Ichimiya Y, Matsuda S, Fujino Y, et al. Profiling of the care processes for laryngeal cancer with the Japanese administrative database. Tohoku J Exp Med. 2011;223:61-6.

16. Tagami T, Matsui H, Fushimi K, et al. Prophylactic Antibiotics may improve outcome in patients with severe burns requiring mechanical ventilation: propensity score analysis of a Japanese nationwide database. Clin Infect Dis. 2016;62:60-6.

17. World Health Organization (WHO). The Asia-pacific perspective: redefining obesity and its treatment. Sydney: Health Communications Australia; 2000.

18. Kim SY, Lim HL, Choi HG. Smoking and alcohol consumption are associated with the increased risk of peritonsillar abscess. Laryngoscope 2020; Feb 10.doi:10.1002/lary.28510 [Online ahead of print].

19. Bagley SC, White $\mathrm{H}$, Golomb BA. Logistic regression in the medical literature: standards for use and reporting, with particular attention to one medical domain. J Cli Epidemiol. 2001;54:979-85.

20. Hasegawa J, Hidaka $H$, Tateda $M$, et al. An analysis of clinical risk factors of deep neck infection. Auris Nasus Larynx. 2011;38:101-7.

21. Celakovsky P, Kalfert D, Tucek L, et al. Deep neck infections: risk factors for mediastinal extension. Eur Arch Otorhinolaryngol. 2014;271:1679-83.

22. Hidaka H, Yamaguchi T, Hasegawa J, et al. Clinical and bacteriological influence of diabetes mellitus on deep neck infection: Systematic review and meta-analysis. Head Neck. 2015;37:1536-46. 
23. Petitpas F, Blancal JP, Mateo J, et al. Factors associated with the mediastinal spread of cervical necrotizing fasciitis. Ann Thorac Surg. 2012;93:234-8.

24. Kanda Y. Investigation of the freely available easy-to-use software 'EZR' for medical statistics. Bone Marrow Transplant. 2013;48:452-8.

25. Huang TT, Liu TC, Chen PR, et al. Deep neck infection: analysis of 185 cases. Head Neck. 2004;26:854-60.

26. Lee YQ, Kanagalingam J. Deep neck abscesses: the Singapore experience. Eur Arch Otorhinolaryngol. 2011;269:1241-9.

27. Noda K, Kodama S, Noda K, et al. Deep neck infection: clinical analysis of 299 cases [in Japanese]. Nippon Jibiinkoka Kaiho (Tokyo). 2010;113:898-906.

28. Okuyama A, Higashi T. Usability of clinical information in discharge summary data in the diagnosis procedure combination survey for cancer patients. Int J Environ Res Public Health. 2020;17:521. doi:10.3390/ijerph17020521.

29. Min HK, Choi YS, Shim YM, et al. Descending necrotizing mediastinitis: a minimally invasive approach using video-assisted thoracoscopic surgery. Ann Thorac Surg. 2004;77:306-10.

30. Bernhard M, Lichtenstern C, Eckmann C, Weigand MA. The early antibiotictherapy in septic patientsmilestone or sticking point? Crit Care 2014 Nov30;18(6):671. doi: 10.1186/s13054-014-0671-1.

31. Mazzella A, Santagata M, Cecere A, et al. Descending Necrotizing Mediastinitis in the Elderly Patients. Open Med (Wars). 2016;11:449-60.

32. Wei $D, B i L, Z h u H$, et al. Less invasive management of deep neck infection and descending necrotizing mediastinitis: a single-center retrospective study. Medicine. 2017;96(15):e6590.

33. Cheepcharoenrat $\mathrm{C}$ : The result of treatment of deep neck infection in patients referred cccording to public health system [published online ahead of print, 2019 Oct 22]. Ear Nose Throat J 2019; 145561319881856. doi:10.1177/0145561319881856.

34. Potter JK, Herford AS, Ellis E 3. Tracheotomy versus endotracheal intubation for airway management in deep neck space infections. J Oral Maxillofac Surg. 2002;60:349-54. rd. .

35. Caccamese JF Jr, Coletti DP. Deep neck infections: clinical considerations in aggressive disease. Oral Maxillofac Surg Clin North Am. 2008;20:367-80.

36. Bhatt YM, Small M. Cervical necrotising fasciitis with descending necrotising mediastinitis. Postgrad Med J. 2011;87:570.

37. Santos Gorjón P, Blanco Pérez P, Morales Martín AC, et al. Deep neck infection: Review of 286 cases. Acta Otorrinolaringol Esp. 2012;63:31-41.

38. Feldmen SA, Deal CW, Urquhart W. Disturbance of swallowing after tracheostomy. Lancet. 1966;1:954-5.

39. Singhal P, Kejriwal N, Lin Z, et al. Optimal surgical management of descending necrotising mediastinitis: our experience and review of literature. Heart Lung Circ. 2008;17:124-8. 
40. Sandner A, Börgermann J. Update on necrotizing mediastinitis: causes, approaches to management, and outcomes. Curr Infect Dis Rep. 2011;13:278-86.

41. Fujiwara K, Koyama S, Fukuhara T, et al. Successful Surgical Treatment for Dysphagia Secondary to Descending Necrotizing Mediastinitis. Yonago Acta Med. 2019;62:253-7.

\section{Figures}

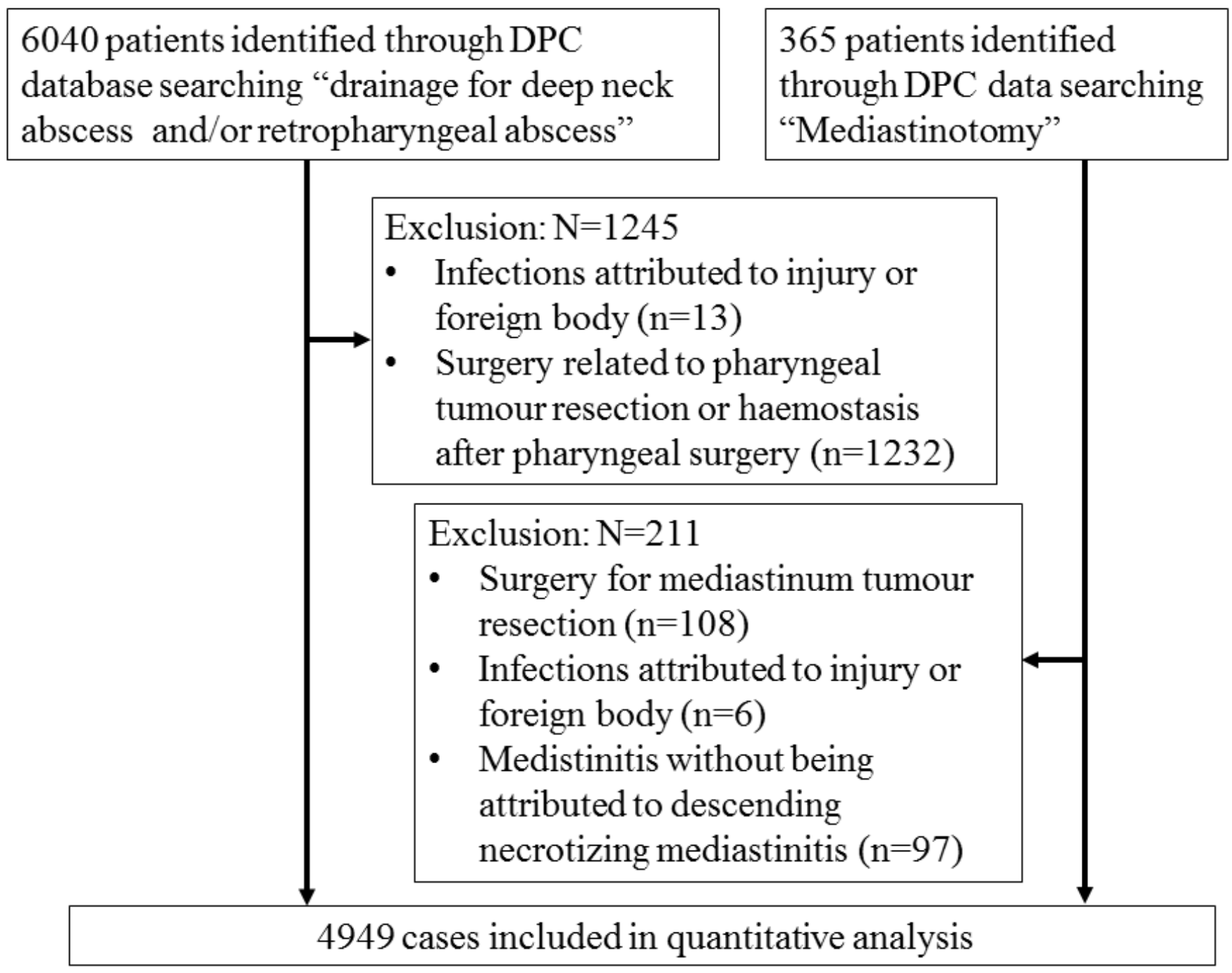

Figure 1

Schematic of patient selection. DPC, Diagnostic Procedure Combination. 


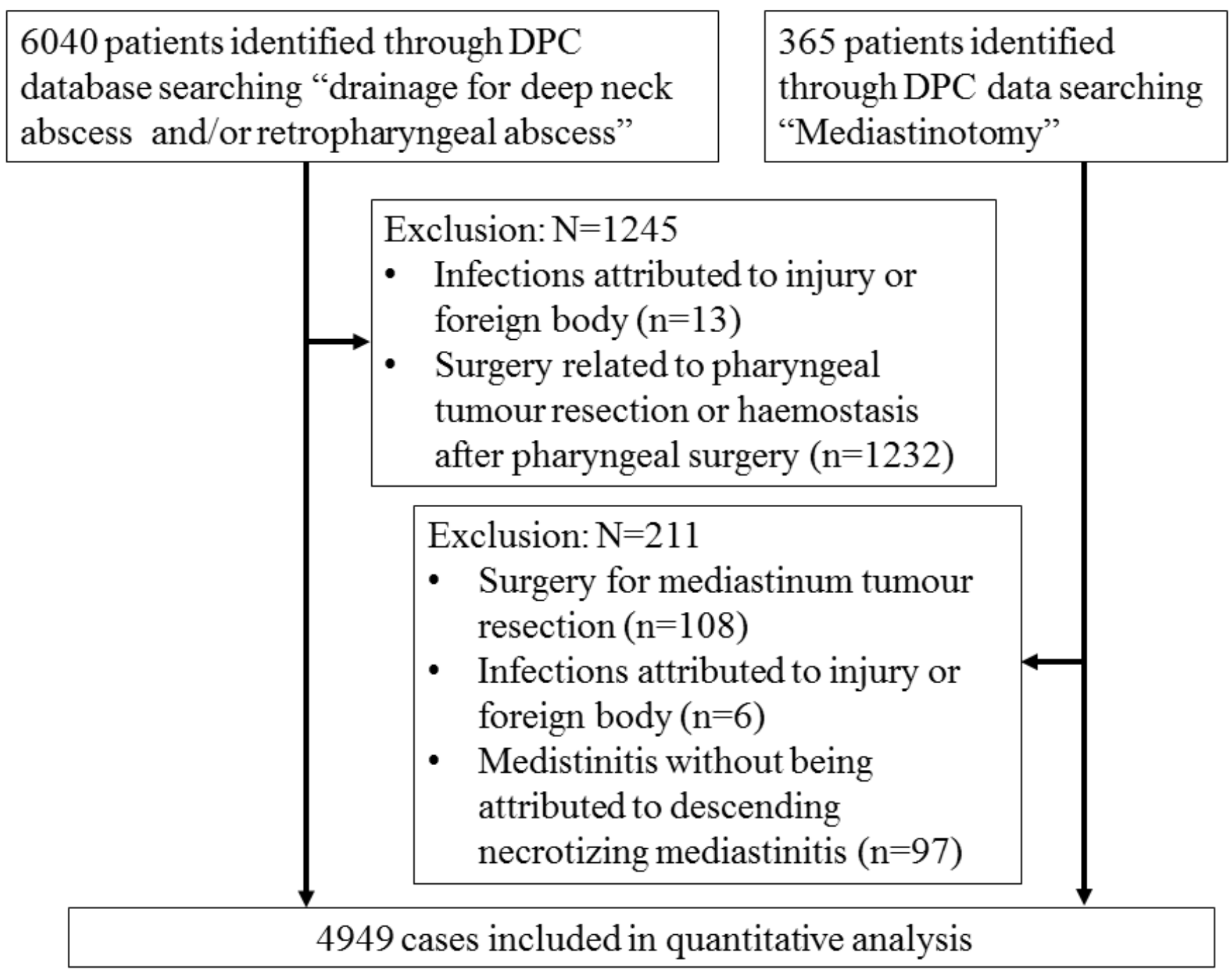

Figure 1

Schematic of patient selection. DPC, Diagnostic Procedure Combination. 